\title{
Information technology transfer and imple- mentation: The introduction of an electronic mail system in a public service organization
}

\author{
Karlheinz Kautz \\ Norwegian Computing Center \\ POBox 114 Blindern, N-0314 Oslo, Norway,Karl.Kautz@nr.no
}

\begin{abstract}
The organizational implementation of information systems is an important and problematic issue of information technology transfer. The case study presented in this paper deals with the introduction of an electronic mail system in a public service organization. An interpretive framework is used to understand the interplay of the different elements which influence the introduction process. It comprises three main components: the content of the introduction, the context in which it takes place, and the social process of the introduction. This involves both a cultural and a political perspective of the process. The analysis of the case is used to present some broader implications for understanding and managing introduction processes.
\end{abstract}

Keywords
Information technology and organization, organizational implementation

\section{INTRODUCTION}

The organizational implementation of information systems, and in a broader sense information technology, has been subject of research for nearly 30 years. Most of the studies relate a set of factors and dependant variables to each other. It has been stated that although this research has produced some valuable information about a number of implementation characteristics, it has only led to little of a unified, coherent body of knowledge (Kwon \& Zmud, 1987).

A demand for research aiming at understanding the complex process of implementation and the linkage between its various elements has been put forward by different researchers (see, $f$. ex,. Markus \& Robey (1988), Wastell (1992)) and a growing number of studies strives for satisfying this request (see Borton \& Brancheau (1994), Montealegre \& Applegate (1994), Walsham \& Waema (1994) for more recent efforts). The study presented here contributes to this line of research. However, while organizational implementation is often considered as a stage in a development process, this article describes and analyses the introduction of a ready-made 
information system purchased on the market. The article deals with the introduction of an electronic mail system in a public service organization. An approval of this technology is highly dependant on reaching a critical mass of users within the different departments of an organization (Marcus, 1990). The paper uses an interpretive framework to understand the interplay of the different elements of this particular introduction process which despite of problems and weakness - at least at the time of writing - has led to a certain acceptance of the system among the employees of the organization.

The remainder of the paper is structured as follows: The next section contains the research background and framework. Section 3 comprises a presentation of the research method. The case description and analysis is included in section 4 . Some broader implications for understanding and managing introduction processes are subject of section 5. The paper finishes with some conclusions in section 6 .

\section{RESEARCH BACKGROUND AND FRAMEWORK}

Research on information technology is performed on the background of different world views. Dahlbom and Mathiassen (1993) see basically two different philosophies which have moulded the field: positivism and hermeneutics. Orlikowski and Baroudi (1991) identify three research philosophies. They distinguish between positivist, interpretivist and critical research. In their survey of contemporary IS research however, they do not find any contributions performed according to the critical paradigm.

Positivistic research is interested in general knowledge, in collecting empirical, unilateral cause-effect laws and factors that can serve as guidelines for action in all situations that seem to be alike. The aim of knowledge is explanation and prediction. This paradigm is the leading one in information technology research. Wastell (1992) points out that such research has produced very little knowledge of apparent benefit to the field. The correlations obtained by rigorous and controlled factor and survey studies contain little divergence and in terms of actual answers, they are often worthless. No real advice for specific work settings can derive from studies seeking general laws.

In hermeneutics, there is an interest in understanding actions by interpreting, not explaining or predicting them. The aim of knowledge is edification and enlightenment. Knowledge is not a general object that can be collected; knowledge is individual. Phenomenological research is based on a view that knowledge of reality is socially constructed by human actors. There is no objective reality that can be discovered by researchers and replicated by others. Different perceptions of reality are ways of making sense of the world. Meanings are shared through an ongoing process of communication and are forms of intersubjectivity rather than of objectivity.

The struggle between positivism and hermeneutics can not be settled. As Dahlbom and Mathiassen (1993) formulate it, it expresses the fundamental and antagonistic difference between technology, the objective, stable, controllable, and people, the subjective, unsteady, and hardly controllable. This might be seen as a simplistic and extreme position, important however is their argument that understanding and respecting the relation between information technology and people is a valuable source for developing and managing information technology in organizations. The introduction of information technology is not just a process of technical change, but also, or first and foremost, means organizational development.

Hirscheim et al. (1987) argue that systems development which aims at the use of computer technology is a social process which relies on technology. Bjerknes (1992) describes this social process as a dialectical reality of mutual reciprocating influences and contradictions in 
which organizations are seen as diversified structures. There, many points of view and interests exist. This makes the introduction of information technology a complex, social and political phenomenon. This political dimension is also stressed by Markus (1983) and Franz and Robey (1984). Pluralistic research is requested. A research that comprises all vital aspects of introducing information technology, namely the technical, the social, the cultural, the organizational, and the political.

Walsham (1993) puts forward such an approach with his interpretive approach to systems development. He advocates research that counterbalances and complements not only positivism and its concerns of technology and technological control, but also hermeneutics and its desire for increased understanding through mere description. He includes issues such as power, control, conflict, and contradictions as raised by critical social theory in his framework. This approach combines elements of interpretivist and critical research as presented by Orlikowski and Baroudi. Walsham has used his framework successfully for studying the development and implementation of computer-based information systems as well as for the formation and implementation of IT strategies.

Here, the framework will be applied to study the introduction of a software system that was purchased ready-made on the market. The framework comprises three main components for understanding organizational change. The content of the modifications is naturally an important element of any change processes. With respect to the organization, this comprises planned alternations of products and services, business process, and formal organizational structures and roles. With respect to the associated information technology hardware, system software, and application software are all constituting parts of an information system that have to be considered. The second component is the context in which the introduction takes place. Elements of this component include the history of previous undertakings in connection with computer-based systems as well as the social relations and the social infrastructure of those involved in and concerned by the information technology. On a broader level the context comprises also the sectorial and national environment of the organization. The third component of the framework touches the social process. It involves both a cultural and a political perspective of the introduction. The cultural perspective takes into account the role and interaction of different subcultures; the political perspective stresses the role of information technology in processes of control and power. The cultural and the political perspective are not independent of each other. In the following description and analysis of the case, emphasis has been put on the dynamic interlinking between these components.

\section{RESEARCH METHOD}

The research presented here is part of a larger project investigating the introduction and use of existing technologies in public service organizations. The researcher himself was involved in the project as a supporting external consultant. The aim of the study is to contribute to a broader understanding of the introduction processes of information technology.

For this purpose case studies are, according to Zmud et al. (1989), highly appropriate, in that they offer a holistic view of the processes involved as well as a high level of richness of worldly realism.Yin (1989) discusses case studies and ethnographic studies. Case studies are structured and purposeful from the start, whereas ethnographic studies are less structured and open. The approach of the study presented here has elements of both types with a tendency towards case studies. Although no explicit hypotheses are stated, the study is based on a clearly expressed perspective. A similar approach is advocated by Carstensen et al. (1995) in their 
research on the work of software testers. The purpose here is to investigate how standard computer-based communication technology is introduced and used in large organizations.

There are disadvantages with a case study oriented approach like the lack of control and a corresponding difficulty in generalizing the results. Knowledge gained through case studies is not formally generalizable, but this does not mean that it does not contribute to the collective body of knowledge of a discipline (cf. Eisenhardt (1989) and Flyvbjerg (1992)). Any research approach is inevitably a trade-off between tightness of control and richness of worldly realism. Here the latter has been chosen, because whilst it may be compromising the ability to generalize, it offers valuable insights about the specific process under discussion.

The research started in summer 1993 and was carried out during a period of 18 months. The study and the data analysis is based on qualitative as well as on quantitative data. The principle method to collect data was semi-structured interviews. In total 12 formal interviews with management personnel, project members and related clerical officers and consultants were conducted. These were supplemented by many informal conversations. The interviews were recorded in writing and a resulting report was acknowledged by the organization. It is known that interviews are verbal reports and as such subject to problems of interview bias, poor memory, and inaccurate statements. Kidder (1981) argues however that many critics tend to exaggerate the significance of interview bias. They overlook the fact that scientists interested in social and organizational issues are dependent upon data which have been collected by means of oral or written reports. The reports are, no matter how collected, invariably subject to essentially the same bias than those based on observations, experiments, or survey questionnaires. One must nevertheless be aware of the fact that interview subjects have a tendency to rationalizing their behaviour and acts. The interview material has therefore been substantiated with document studies. Strategy documents, internal reports and memos as well as the organization's internal newspaper served as additional sources. Finally a survey concerning the use of the system was performed among all employees of the headquarters half a year after the system was technically available for everyone. This survey had a high response rate as half of the employees answered the questionnaire.

\section{THE CASE DESCRIPTION AND ANALYSIS}

Subject of the case study are the headquarters of a public service organization which delivers communication services to the citizens of Norway. After a reorganization the headquarters now consist of 8 business departments and 10 accompanying service centres. In total 600 people are employed there, the majority are clerical officers, consultants, and case workers.

In 1992 a project group consisting of 3 full time project members was established in the support department for technical services and internal administration to introduce electronic mail into the organization. In line with the organization's official strategic document, both top management and the project leader stated that the project pursues two aims. These are (1) to increase the efficiency of the organization by improving office work, and (2) to gather experience with electronic mail (email) as a basis for the development of new customer services. The group's assignment was to take care of the necessary infrastructure, to supply system software and to install the email system for the employees of the headquarters. Along with the technical tasks, the group is responsible for providing information about the system and to prepare training material.

In 1993 much of the product-independent infrastructure work had been finished, but no decision had been made about the email system to be purchased. This was due to discussion about a common system with the two subsidiaries of the main organization which also were planning 
to use email. Finally after negotiations which did not lead to a common solution an email system was bought for the headquarters. In May 1994 it was announced in the organization's newspaper that all employees of the headquarters had access to the new email system. At this point in time the project was half a year delayed with regard to the original time schedule and it was only then when all employees were officially informed about the introduction of the system. Furthermore, no formal training was provided. Besides a very short on-line document explaining the system's main features, a booklet was distributed that should serve as a user manual.

The user survey half a year later showed that $90 \%$ of those who answered the survey used the system. A third of them considered themselves as people who utilized the system to a great extent and 50\% considered the system as beneficial for their work. On the other hand, also 50\% mentioned that they had problems with the system and that they did not know enough about it to really take advantage of all its possibilities.

\subsection{Contextual aspects of the case}

According to market surveys during the last years the organization is ranked among the most popular in the country concerning customers' satisfaction with the services delivered. In parts of its business the organization enjoys a monopoly on the market. However, lately, the organization has been exposed to growing competition, a trend which probably will increase in the future.To meet this challenge and to become more market-oriented, the headquarters have undergone a reorganization process in 1993 which ended up in the above described organizational structure. One effect of this process is that departments and service centres have to work more result-oriented. They have to sell their services to each other, which also means that they have to compete with other providers on the open market.

A momentum which is more directly related to the email project, is its origin. The idea of introducing electronic mail into the organization goes back to 1990 to a technical consultant who worked in the then existing computer department of the organization. At that time however his ideas were not severely considered. An event of some significance is an ensuing attempt to introduce email rather informally. The system chosen was regarded as very user-unfriendly and technically unstable. What counts more, however, is the fact that it was mainly used to send around long circular documents which were not of direct interest for daily work. In general the number of messages was very low and only few employees looked into their post boxes every day on a regular basis. Many employees did not even know that they could send messages themselves, they thought that the system was a tool for management only to distribute notes and information. All this lead to a situation were the system was not used very actively and the introduction was widely considered to have failed.

Finally in connection with the new departmentalization of the headquarters it is worth mentioning that the project leader who had held a leading position in the technical support department when the project idea emerged, left this department short after the official start of the project to join a more traditional business-oriented department and took the project with him.

All these features had an important influence on the direction of events the project took in the course of time. Further contextual features are closely related to culture in the organization and will be taken up in the subsequent section. 


\subsection{A cultural view at the introduction process}

The organizations culture as a whole is moulded by the original product which the organization provides, namely the delivery of communication services. The predominance of this culture is mirrored by the fact that the email project was moved to the department which develops products for the national information exchange market and not to the department which has as its task to develop and maintain physical and electronic net services and the internal infrastructure of the organization. Management in the product-oriented department expressed that they like to control the speed of the introduction such that email on the commercial market does not outlive the traditional products too fast.

Another characteristic of the culture is its technique-orientation. The employees are open for technical innovation and many new technical systems have been introduced into the organization in the past. This openness provided the ground for acceptance of the email system. The technical orientation has however also apparent disadvantages. Success of the project is measured in number of installations. It has not yet been monitored, and it is so far not planned to do so, whether the project is reaching its aims, to increase efficiency and to gain new knowledge.

The organization is hierarchically structured. Work, officially, has to be performed following formal rules. This leads to a diffuse picture concerning the informal and formal communication patterns. As no directive has been issued, it is not clear whether, and for which tasks, the use of the email system is mandatory or optional. Today, the system is not used to exchange documents as the employees are insecure about its formal status. They wait for official rules for the use of the system with the effect that one important potential of the system is not exploited at all.

Hierarchical thinking and rigid communication patterns had direct consequences for the cooperation in the project. The project group informally notified the user support group and the training center about a need to include them in the project. None of the groups, which are both understaffed, reacted to the informal request. They waited for an official call while the project group was waiting for being contacted by them.

However, besides the formal communication channels, informal ones exist which are viable for the organization. A good example here is user support's network of key persons in all departments who actively support the introduction of information technology. Although no direct evidence can be presented for the introduction of the email system, the assumption can be made that this informal network also worked in this case.

Hierarchical thought and the idea of centralisation is also expressed in management's attitude towards the project. By and large, management believes that to get the employees to adopt the system it is sufficient that they themselves use it. The project has only been paid little attention and only comparable little resources have been provided. Management's aims with the introduction have not been communicated to the employees clearly and for a long time uncertainty prevailed whether the introduction had more the character of a technical experiment or whether a new support for daily work was provided. Both aims are not contradictory, but as mentioned above they have not been explained enough.

Another attribute of the culture is the fact that many of the employees have spent their whole professional life in the organization. This leads to a enormous loyalty, a strong corporate identity, and an esprit de corps concerning the values the organization stands for. One example for loyalty towards the own department has been described earlier. Another instance exhibiting the relationship between loyalty and communication can be presented here. The project team did not want to jeopardize the organization's image in the public and the project leader, by all means, wants to avoid another failed introduction. Therefore the group did not inform the em- 
ployees about the introduction before they were fairly certain about the technical stability of the system. This, however did not only have positive effects. It also contributed to rumours and uneasiness concerning the change of working routines in the headquarters.

The esprit de corps shows to be important when the settled distinction between those who are "internal", who have spent their working life in the organization, and those who have been hired from the outside is considered. This is manifested when a look at the role of two external consultants in the project is taken. The consultants are welcome as a reinforcement of the approach chosen by the project group, but their proposals are only taken up hesitantly and they are more or less confined to a passive role. Another example in this respect is the way the ideas of a manager who was recruited from the outside are treated. His plan to integrate email with other office systems was met with resistance. It was interpreted as an attempt to change working routines with a "big bang" approach and responded with a suggestion to start with a smaller stand-alone solution. Integration however is not conflicting to a stand-alone start given that an incremental approach and a design are chosen which take into account future extensions.

Besides the cultural-associated scepticism towards the outside proposal, this example also has a political dimension pointing to a power struggle between a top manager and middle management. This will be described in more detail in the following section.

\subsection{Some political elements of the introduction process}

The future of the email project is subject to ongoing discussions in the headquarters. The debate about a stand-alone or an integrated solution has led to some delays and some uncertainties about the further course of the work. The considerations are not only about an organizational and a technical approach to the utilization of technology. They also have a political momentum.

This momentum is concerned with the control over the project. One possible consequence of following the top manager's proposal to strive for integration will be a loss of control for the actual project leader. The project team might be changed and experts on office work in general as well as experts on existing office support systems might be included. Ultimatively the leadership of the project might be placed into hands of a person who is more sympathetic to the ideas of this top manager.

Besides a power struggle between top management and middle management, another interdepartmental conflict can be uncovered here. The mentioned top manager also wants to move the project away from the product, communication service-oriented department. As explained before, until now the project lies in the responsibility of this traditional department where management wants to control the introduction of email to protect its own products. In this department top management shows little understanding that email is a supplement, and not a replacement of existing products. In addition, not much sense can be found here for the possible benefits of email for internal work. Moving the project to another department and going for an integrative solution will change the power relationship in the headquarters which historically was dominated by the product-oriented department. The conflict has not been settled yet and negotiations are still going on.

Another example how the introduction process has been used politically, can be demonstrated by the cooperation problems between user support and the project team. User support has been understaffed. Not reacting upon the project teams' request for help, gave them another opportunity to demonstrate their personal situation with the aim to get additional staff.

But also on a higher organizational level political processes are involved: the decision about what email system to purchase by the headquarters and two of the organization's subsidiaries, was on the surface a debate about opting for a local product which is in line with a chosen stand- 
ard or a product from an international vendor which fits into a larger product line. The technical argument that no interface between these systems exists is only of minor significance, because the development of such a gateway is not a big technical challenge. The discussion is much more about who is controlling the whole organization. The two comparably new subsidiaries who operate on the financial market are contributing the major part to the organization's financial success, whereas the original parts of the organization which still control the organization are financially less profitable.This control is now challenged in decision making processes like the described one. For the introduction process under consideration, no compromise could be found. Different systems have been bought, further delays occurred and lately the two subsidiaries have been separated from the mother organization and operate now independently.

\section{IMPLICATIONS FOR IT INTRODUCTION AND TRANSFER}

A discussion which aims at some more wide-ranging implications for the introduction of information technology has to take into account to what extent the described case presents a success story or a failure. This is however not such a simple task as different success criteria may be applied.

The introduction of the email system can be considered a success as the system in the initial phase is used by approximately $40 \%$ of all employees and the majority of them experience the system to be beneficial for their work. Yet, the project has not been finished within its original time constraints and many people report problems with the use of the system. In this respect, the project has not been successful. No judgement can be made whether the project is a success or a failure in relation to the objectives pursued by senior management. Nonetheless, it is important to note that the introduction process has at least partly been performed as a project that was mastered in a controlled way, in contrast to other introduction processes which evolve totally uncontrolled. Some disagreements like the political considerations described which were related to the selection of the email system just need time to be settled or resolved. Other obstructions might be cleared out of the way when handled appropriately. Therefore, in the following a closer look is taken at some specific aspects of the case presented.

\section{Project mission and project goal.}

According to the majority of senior management and the project leader, the introduction of the email system has two goals. On the one hand, an aim is to carry out office work more productively. As possible gains, more efficient communication and saving man power were named. The other aim is to learn about the technology for the development of future products for the market. As mentioned, these aims are in line with the official strategy of the organization.

These are strong and unambiguous objectives which are clearly related to the business needs of the organization. They are not determined by short-sided and seasonal demands, but by enormous and pressing requests in a competitive situation, but in a long-term perspective. Such a context has shown to be stipulating for the introduction of technology in other areas (cf., Roberts \& Barrar, 1992).

However, it is unclear in how far these aims are known in the headquarters. They have not been communicated to the employees. Communication between the directly involved groups and general information seems to be a problem in this project. The misunderstandings resulting from this situation make it difficult to introduce and promote technology which is supposed to support integration, coordination, and cooperation. The embodiment of the project within the 
organization is questionable and the role the technology should play in the organization is not obvious for the employees either. This resulted in an uncertainty and a hestitant and limited utilisation of the system.

Little can be said whether the mission has been accomplished. No measurement programme concerning increased efficiency and a higher level of knowledge has been initiated. The basic lesson learned here can be formulated like this: Defining clear goals is not enough, they have to be communicated and their accomplishment has to be monitored and evaluated.

\section{Senior management involvement and support.}

Senior management's attitude towards the introduction of email gives no uniform picture in the headquarters. The spectrum ranges from fear of a competing product and doubts about the usefulness of the system to absolute enthusiasm about the operation and the possibilities of the technology.

Most of the management shows an enormous interest in using the system themselves and are convinced about its benefits for their work, but also for the organization as a whole. Within this group, no consensus has been reached so far how to combine email with the existing technical office environment. Integration versus stand-alone are the alternatives discussed among the leaders.

There is a consensus in the group that the most important support they can provide for the introduction process is that they themselves use the system and nearly all of them describe themselves as eager users. There is little doubt that such symbolic acts have some significance for the introduction process. It is more likely that the employees will not use a new communication channel which is supposed to support information exchange between all groups, if management does not use it.

But besides this contribution management's effort for supporting the introduction process has to be characterized as quite passive. Management did not appear really committed. Sound engagement like active participation in introduction arrangements and courses or public appeals motivating the usage of the system have not taken place. Support in form of provision of resources has been fairly modest. Management was not willing to participate in a proposed extended project group 'organizational introduction' and were quite hesitant in providing personnel for such a group. Some of them thought the group to be a good idea, but did not want to delegate staff from their own department. Extra resources for training where not considered at all. Here lies a reason why the introduction process struggled for a while and for the uncertainty about the system's purpose and the problem's of use which were reported.

We get another confirmation that mere interest and passive involvement is not sufficient when it comes to senior management 's role in change processes in the context of technology introduction. Lack of support can slow down and jeopardise the process, but in the presented case, so far utilisation has not stopped or faded away because a technology-friendly community has created the critical mass which is necessary for the meaningful exploitation of a communication medium.

\section{Education and training.}

The headquarters have an own consultancy and competence center. Two persons there are responsible for organizing education related to the use of information technology. A detailed procedure exists for arranging training which can be adjusted to specific events like the introduction of an email system. The IT training consultants partly prepare courses and hand- 
books themselves, partly they take care of hiring lecturers and buying courses on the market.

One of them was quite early informally notified about the email project. Formally their support was requested very late and in a very restricted form. They were never officially members of the project group.

The organization got hold of the original course material issued by the email supplier. This material comprised a one day tutorial with exercises for every major feature of the tool. The project group published however only a one page on-line help and used the competence center only to produce a small brochure explaining the most important functions of the system. This brochure presented a subset of the original documentation and no exercise tasks were included. No formal training was provided.

More than half of those employees who reported problems with the system argued that this was due to a lack of training in combination with a lack of time for self-study. The more general message here is that formal education and training which go beyond studying a handbook and provide hand-on use experience are important components of a successful introduction strategy.

\section{Organizational validity and usefulness.}

The headquarters are part of a formal department where work to a large extent is rule-governed. Half of the user survey respondents agree that email is a significant tool for the organization. Only 10 employees disagree strongly. A third of the staff who answered consider themselves as intensive users, many report that the system makes it easier to exchange messages and they state that it has replaced many yellow stickers. Some say that they save many telephone calls now.

Concerning document exchange the situation is more complicated. Although again half of the respondents think that email is useful for the exchange of documents, $25 \%$ share the opinion that there are many documents which cannot be sent by email and only few formal letters and notes have been replaced by email transmissions.

One reason why the email is less applied than desired lies in the fact that there has been little information about the use of the system. There exists a lack of guidelines about what should be sent and to whom. The established steering mechanism and the reporting rules concerning formal management and the treatment of cases are violated by the system. There is no possibility to properly trace a document through the organization and so far no announcement has been made whether email documents are considered as official documents. One respondent even meant that files which have been sent by email are neglected and handling them is more often postponed than the work with physically received papers.

In general it is felt in the organization that the work procedures and regulations and the technical support are not adjusted to each other. This is regarded as a major obstacle for a more far reaching utilisation of the email system.

This leads to a broader implication: The information technology to be introduced has to fit the structure of the organization and the work patterns of the departments, groups, and individuals. If this is not the case, the necessary adaptation should be part of the introduction process.

\section{CONCLUSIONS}

The presented case describes the introduction of an email systems. It demonstrates the relationship between organizational change and technology transfer. Despite a number of problems, it 
looks as if in this case the technology is on the way of being accepted and used by the staff of the organization.

The interpretive framework which was used broadened the analysis and comprised not only the technical content of the email system to be implemented, but also its context and the dynamics of the introduction process. Such a perspective uncovers cultural and political elements. These are interdependent and influence the transfer of the technology.

On this basis four broader implications for the introduction of information technology were discussed. These concerned the definition, communication and monitoring of project goals, the role of senior management and education and training, and the relationship between organizational structure and work patterns and information technology.

The case appears to be quite typical and does not provide many surprises and new theoretical insights. It is rather a further piece of evidence for the usefulness of the interpretive approach to understand the implementation of information technology as a social process. Introduction of standard, computer-based communication technology, in this respect does not differ more tailor-made information systems.

Although the broader implications might not offer much novelty, they can be put into concrete terms. This concretization can be of benefit for the organization in question as the introduction process is not finished yet.

A project group "organizational introduction" still seems appropriate. Such a group needs real support by top management, both in form of resources, but also by active participation.

Besides management and technical-oriented staff, the involved units user support and training should take part as well as delegates from the clerical officers and case workers from different departments.

The group should

- use the existing communication channels like the organization's new magazine or the email system itself to explain the project

- establish a measurement scheme to monitor the accomplishment of project goals by e.g. recording

- the labour hours used on work cases

- the number of telephone calls and paper-based messages

- the number and degree of customer satisfaction

- the number of ideas for new products

- develop or initiate the development of procedures and regulations for the use of the technology for reporting and official document handling

- provide for short training courses and comprehensive paper-based and on-line manuals.

These recommendations do not offer a guarantee for success, but they have some value in that may help minimise the risk of failure for the introduction of information technology and in that they can serve as a starting point for the formulation of introduction strategies in other technology transfer projects.

Finally, the question remains, why, if we accept that these guidelines are fundamental, does it still occur that introduction of information technology happens the way as described in this case: If this "knowledge" is not known, the question is, why is this so; and if it is known, the question is, why is it not applied? These are questions open to further investigation in technology or to be more precise knowledge transfer research. 


\section{REFERENCES}

Bjerknes, G., (1992). Dialectical Reflection in Information Systems Development. In Scandinavian Journal of Information Systems, Vol. 4, pp. 55-78.

Borton, J. M., J. C. Brancheau, (1994). Does an Effective Information Technology Implementation Process Guarantee Success?. In Levine, L. (ed.), Proceedings of the IFIP TC8 Working Conference on Diffusion, Transfer and Implementation of Information Technology, Pittsburgh, PA, USA, 11-13 October, 1993, North Holland, Amsterdam, London, New York, pp. 159-178.

Carstensen, P. H., C. Sørensen, T. Tuikka, (1995). Let's Talk about Bugs. In Scandinavian Journal of Information Systems, Vol. 7, No. 1, pp. 33-53.

Dahlbom, B. \& L. Mathiassen, (1993). Computers in Context - The Philosophy and Practice of Systems Design. Cambridge \& Oxford:NCC Blackwell.

Eisenhardt, K. M., (1989). Building Theories form Case Study Research. In Academy of Management Research Review, Vol. 14, No. 4, pp. 532-550.

Flyvbjerg, B., (1992). Rationality and Power - The Science of the Concrete (In Danish). Akademisk Forlag. Købnhavn.

Franz, C. R., D. Robey (1984). An Investigation of user-led System Design: Rational and Political Perspective. In Communications of the ACM, 27(12):1202-1209.

Hirschheim, R., H. Klein, M. Newman, (1987). A Social Action Perspective of Information Systems Development. In DeGross, J. and C. Kriebel, eds., Proceedings of the 8th International Conference on Information Systems, pages 45-56.

Kidder, L. H., (1981). Selltiz, Wrightman \& Cook’s Research Methods in Social Relations. Holt, Rinehart and Winston, New York.

Kwon, T. H., R. W. Zmud, (1987). Unifying the Fragmented Models of Information Systems Implementation,. In Boland, R. J., R. A. Hirschheim (eds.), Critical Issues in Information Systems Research, Wiley \& Sons, New York.Winston, New York, pp. 227-251.

Markus, M. L., (1983). Power, Politics, and MIS Implementation. In Communications of the ACM, 26(6):430-444.

Markus, M. L., (1990). Toward a "critical mass" theory of interactive media. In Fulk, J., C. Steinfield (eds.), Organizations and Communication Technology, Sage Publications. Newbury Park, Calif., USA, pp. 194-218.

Markus, M. L., D. Robey, (1988). Information Technology and Organization Change: Causal Structure in Theory and Practice. In Management Science, Vol. 34, May 1988, pp. 583-598. 
Montealegre, M., L. M. Applegate,(1994). Information Technology \& Organization Change: Lessons from a Less-Developed Country. In Levine, L. (ed.), Proceedings of the IFIP TC8 Working Conference on Diffusion, Transfer and Implementation of Information Technology,Pittsburgh, PA, USA, 11-13 October, 1993, North Holland, Amsterdam, London, New York, pp. 99-131.

Orlikowski, W. L., J. J. Baroudi, (1991). Studying Information Technology in Organizations: Research Approaches and Assumptions. In Information Systems research, Vol. 2., No.1, pp. 128 .

Roberts, H. J. and P. R. N. Barrar, (1992). MRPII implementation: Key Factors for Success. In Computer Integrated Manufacturing Systems. 5(1): 31-38.

Walsham, G., (1993). Interpreting Information Systems in Organizations.Wiley Series on Information Systems, Chicester.

Walsham, G., T. Waema, (1994). Information Systems Strategy and Implementation: A Case Study of a Building Society. In ACM Transactions on Information Systems, Vol.12, No.2, pp. 150-173.

Wastell, D. G., (1992). The Social Dynamics of Systems Development: Conflict, Change and Organizational Politics. In S. Easterbrook, S., (ed)., CSCW: Cooperation and Conflict?. Springer Verlag, London, pp 69-91.

Yin, R. K., (1989). Research Design Issues in Using the Case Study Method to Study Management Information Systems. In Cash, J. I., P. R. Lawrence (eds.), The Information Systems research Challenge: Qualitative Research Methods. Vol. 1. Havard Business School Research Colloqium, pp. 1-6.

Zmud, R., M. H. Olson, R. Hauser, (1989). Field Experimentation in MIS Research. In Benbasat, I., ed., The Information Systems Research Challenge: Experimental Research Methods, Vol. 2, Harvard Business School Research Colloquium, pp. 97-112.

\section{BIOGRAPHY}

Karlheinz Kautz is employed as a research scientist at the Norwegian Computing Center. Before joining this research institute he was a researcher and lecturer at universities in Germany, England, and Norway. Kautz's education includes a MSc in computer science from the Technical University of Berlin, Germany and a doctorate in systems development from the University of Oslo, Norway. He is vice-chair of IFIP TC 8 WG 8.6 on Diffusion, Transfer, and Implementation of Information Technology and a member of ACM. He has published in Information Technology \& People, Information and Software Technology, and the Scandinavian Journal of Information Systems and co- authored a book on prototyping. His research interests are in technology transfer, organizational impacts of information technology, evolutionary systems development, participatory design, prototyping, software quality and software process improvement. 